\title{
Survival Analysis of Patients with Thoracic Duct Ligation during Thoracoscopic Esophagectomy
}

\author{
Rui-feng Yang ${ }^{1}$, Shu-jing Sui ${ }^{2}$, Lei-lei Yu ${ }^{3}$, Hong-yan $\mathrm{Li}^{4}$, Run-qi Zhang ${ }^{1}$ and Peng Wang ${ }^{1}$ \\ ${ }^{1}$ Department of Thoracic Surgery, Tai'an City Central Hospital, Tai'an, Shandong, China \\ ${ }^{2}$ Department of Gastroenterology, Tai'an City Central Hospital, Tai'an, Shandong, China \\ ${ }^{3}$ Department of Endocrine, Tai'an City Central Hospital, Tai'an, Shandong, China \\ ${ }^{4}$ Department of Anesthesiology, Tai'an City Central Hospital, Tai'an, Shandong, China
}

\begin{abstract}
Objective: To determinate the effect of thoracic duct ligation during thoracoscopic esophagectomy on esophageal cancer patients survival.

Study Design: A descriptive study.

Place and Duration of Study: Tai'an City Central Hospital, Tai'an, Shandong, China, from June, 2016 to June, 2021.

Methodology: All cT1b-3N0M0 stage esophageal cancer patients were randomly divided into thoracic duct ligation group and non-ligation group. In addition to thoracoscopic esophagectomy, thoracic duct ligation was also performed in the experimental group. The general data of two groups were compared by Chi-square test, with statistical significance at $p<0.05$. The effect of thoracic duct ligation on disease-free survival (DFS) and overall survival (OS) was analysed by Kaplan-Meier and Cox regression.

Result: There was no significant difference in gender, age, tumor location, depth of invasion, degree of differentiation and presence of tumor thrombus between the ligation group (33 cases, 47.8\%), and the non-ligation group (36 cases, $52.2 \%$ ). Cox regression analysis showed that depth of invasion $(p=0.0014)$, degree of differentiation $(p=0.0036)$, presence of tumor thrombus ( $p$ $=0.0367)$ and thoracic duct ligation $(p=0.0057)$ were independent factors affecting DFS. Meanwhile, the depth of invasion ( $p$ $<0.0001)$, presence of tumor thrombus $(p=0.0073)$ and age $(p=0.0129)$ were independent factors affecting OS.

Conclusion: Thoracic duct ligation during thoracoscopic esophagectomy can affect DFS in patients with pT1b-3N0M0 esophageal squamous cell carcinoma, and the thoracic duct ligation, depth of invasion, degree of differentiation and presence of tumor thrombus are independent factors. Meanwhile, the depth of invasion, presence of tumor thrombus and age were independent factors affecting OS.
\end{abstract}

Key Words: Esophageal cancer, VATS, Esophagectomy, Thoracic duct ligation, DFS, OS.

How to cite this article: Yang RF, Sui SJ, Yu LL, Li HY, Zhang RQ, Wang P. Survival Analysis of Patients with Thoracic Duct Ligation during Thoracoscopic Esophagectomy. J Coll Physicians Surg Pak 2022; 32(03):288-292.

\section{INTRODUCTION}

The immunologists, honoured with the 2018 award in physiology or medicine, pioneered immunotherapy, which harnesses the body's immune system to fight cancer and achieved great success. ${ }^{1,2}$ There have been different opinions on whether to preventively ligate the thoracic duct during esophagectomy. ${ }^{3-5}$ With the development of medicine, video assisted thoracic surgery (VATS) has gradually become the mainstream surgical method for the treatment of early- and middle-stage of esophageal cancer due to its advantages of small trauma and rapid recovery. ${ }^{6}$

Correspondence to: Rui-feng Yang, Department of

Thoracic Surgery, Tai'an City Central Hospital, Tai'an,

Shandong, China

E-mail: xwk_yrf@163.com

Received: August 23, 2021; Revised: October 04, 2021;

Accepted: December 29, 2021

DOI: https://doi.org/10.29271/jcpsp.2022.03.288
Under the new surgical method, there is still need to consider whether the thoracic duct should be ligated. Previous studies have shown that the prophylactic ligation of thoracic duct during thoracoscopic esophagectomy can affect the early postoperative absorption function and immune function, especially the decrease of CD4+ T cells. ${ }^{7,8}$ So whether the ligation of thoracic duct will cause serious damage to the immune system, thus affecting the OS and DFS is a subject worthy of study.

The aim of this study was to analyse the survival of patients undergoing thoracic duct ligation with esophageal cancer.

\section{METHODOLOGY}

Patients with esophageal cancer admitted to Tai'an City Central Hospital, Tai'an, Shandong, China, from June, 2016 to June, 2021. Squamous cell carcinoma patients with $\mathrm{Tlb}-3$ invasion, confirmed by endoscopic ultrasonography and biopsy, were enrolled. Patients with distant metastasisand lymph node metastasis detected by PET CT, those with serious cardiopulmonary and immune diseases, and postoperative pathology beyond T1b-3NOM0 stage, were excluded from the study. The general 
condition was evaluated by Doppler echocardiography, lung function, liver and kidney function, blood routine coagulation and so on. The study was approved by the Committee on Medical Ethics of the Hospital, and written informed consent was obtained from all patients.

All patients underwent thoracoscopic and laparoscopic esophagectomy with cervical mechanical anastomosis by the same group of thoracic surgeons. All diagnoses and treatments were carried out in strict accordance with NCCN Clinical Practice Guidelines in Oncology. ${ }^{9 \cdot 12}$ To identify thoracic duct easily, patients were advised to drink 100-150 ml olive oil 8-12h before operation. ${ }^{13}$ During operation, the azygos arch were ligated and cut off to make it easier to free the esophagus and clean the lymph nodes. Behind lower segment of esophagus, the thoracic duct can be recognised easily and separated between the descending aorta and the azygos vein. The patients were randomly assigned to the experimental group need to ligate the thoracic duct, after the thoracic esophagus was completely free. In order to minimise the influence of technique factors, a single gastroenterologist conducted gastroscopy and endoscopic ultrasound grading, and a single anesthesiologist performed intraoperative anesthesia and vital signs monitoring .

The studied variables included age $(\geq 60,<60)$, gender (male, female), T stage (T1b, T2, T3), differentiation (G1, G2, G3), tumor location (upper, middle, lower), thoracic duct ligation (yes or no), and vascular cancer embolus (yes or no). All patients were reexamined every three months in two years, every six months in five years and every year after five years. ${ }^{14}$ The follow-up deadline was June 16 2021. The follow-up examination item included blood routine, gastroscope, chest CT (plain scan or enhanced scan), neck and abdominal color Doppler ultrasound, etc. PET$\mathrm{CT}$, bone scan, MRI, etc. were supplemented according to the patients' symptoms, signs and routine examination. OS (status $=1$ fordeath, 0 forcensored) was measured from the date ofoperation to the date of death or last follow-up and censored at the last contact date in surviving patients. DFS (status $=1$ for tumor recurrence or metastasis, 0 for censored) was measured from the date of operation to the date of first evidence of relapse or death as a result of any cause, which ever was observed first. For patients who had not relapsed or died, DFS was censored at the last date that the absence of relapse was confirmed.

Chi-square test was used to compare the differences for categorical variables. OS and DFS curves were calculated by the Kaplan-Meier method and compared by the unstratified log-rank test. ACox regression model with stepwise selection was used for the multivariate analyses. Statistical significance was assumed for $p$-value of less than 0.05 . All statistical analyses were performed using SAS 9.2 software.

\section{RESULTS}

After early screening, a total of 71 patients were enrolled in the group. In the ligation group, one case each of anastomotic leakage and severe pulmonary infection were excluded. No chylothorax occurred in both groups. Finally, 69 patients entered the study and completed the follow-up. There was no significant difference in gender, age, tumor location, depth of invasion, degree of differentiation and presence of tumor thrombus between the thoracic duct ligation group (33 cases) and the non-ligation group ( 36 cases) $(p>0.05)$. The results are shown in Tablel.

Table I: Comparison of clinical data between ligation group and control group.

\begin{tabular}{|c|c|c|c|c|}
\hline Data & Classification & PLG $(n, \%)$ & NPLG $(n, \%)$ & $\mathbf{p}$ \\
\hline \multirow{2}{*}{ Age } & $\geq 60$ & 19(51.4) & 18(48.6) & \multirow{2}{*}{0.5285} \\
\hline & $<60$ & $14(43.7)$ & $18(56.3)$ & \\
\hline \multirow{2}{*}{ Gender } & Male & $28(49.1)$ & $29(50.9)$ & \multirow{2}{*}{0.6384} \\
\hline & Female & $5(41.7)$ & $7(58.3)$ & \\
\hline \multirow{3}{*}{ Location } & Up & $5(45.5)$ & $6(54.5)$ & \multirow{3}{*}{0.6886} \\
\hline & Middle & $18(52.9)$ & $16(47.1)$ & \\
\hline & Down & 10(41.7) & $14(58.3)$ & \\
\hline \multirow{3}{*}{ Invasion depth } & Tlb & $17(51.5)$ & $16(48.5)$ & \multirow{3}{*}{0.8319} \\
\hline & $\mathrm{T} 2$ & $10(45.5)$ & $12(54.5)$ & \\
\hline & T3 & $6(42.9)$ & $8(57.1)$ & \\
\hline \multirow{3}{*}{ Differentiation } & G1 & $7(50.0)$ & $7(50.0)$ & \multirow{3}{*}{0.6832} \\
\hline & G2 & $17(43.6)$ & $22(56.4)$ & \\
\hline & G3 & $9(56.2)$ & $7(43.8)$ & \\
\hline \multirow{2}{*}{ Cancer thrombus } & Yes & $7(46.7)$ & $8(53.3)$ & \multirow{2}{*}{0.9191} \\
\hline & No & $26(48.1)$ & $28(51.9)$ & \\
\hline
\end{tabular}
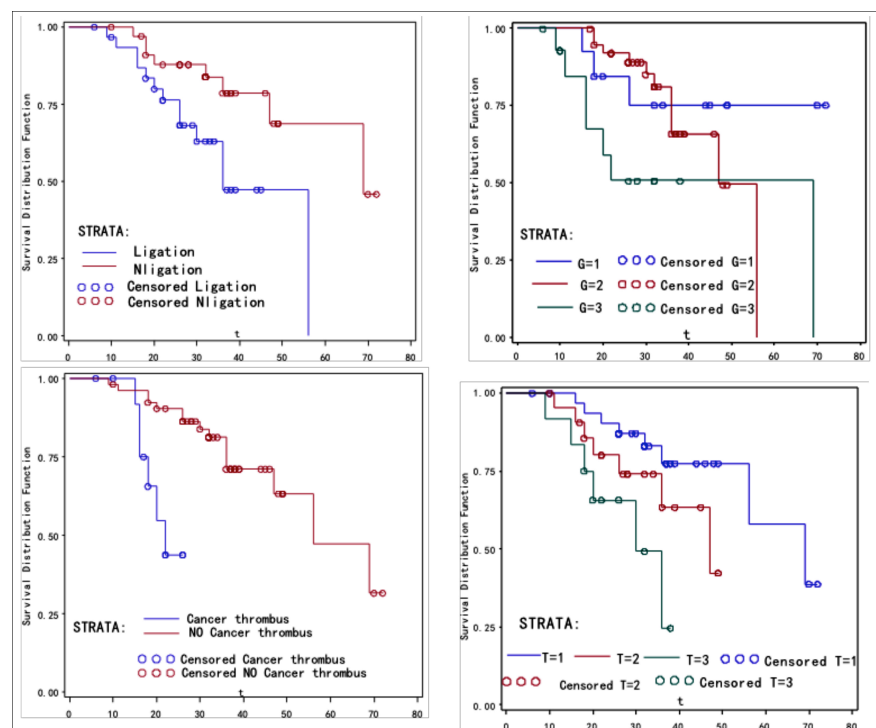

Figure 1: Single factor analysis of the effect of T stage, tumor differentiation, thoracic duct ligation and vasculartumorthrombus on DFS.

DFS was calculated and plotted with the Kaplan-Meier method as shown in Table II and Figure 1. The results showed that gender, age and tumor location had no significant effect on DFS $(p>0.05)$, but depth of invasion, degree of differentiation, presence of tumor thrombus and thoracic duct ligation had significanteffect on DFS $(p<0.05)$.

Cox proportional hazard models for DFS was built for those prognostic factors with $p<0.1$ in the univariate analysis as shown in Table III-a. The result showed that invasion depth $[\mathrm{HR}=2.495,95 \% \mathrm{Cl}(1.426,4.366), \mathrm{p}=0.0014]$, tumor differentiation $[\mathrm{HR}=2.964,95 \% \mathrm{Cl}(1.426,6.158), \mathrm{p}=0.0036]$, vascular cancer embolus [ $\mathrm{HR}=3.385,95 \% \mathrm{Cl}(1.078,10.626), \mathrm{p}=0.0367]$, and thoracic duct ligation $[\mathrm{HR}=4.088,95 \% \mathrm{Cl}(1.506,11.099)$, 
$p=0.0057]$ were the independent risk factors affecting the DFS.

OS was calculated and plotted with the Kaplan-Meier method as were shown in Table II and Figure 2 . The results showed that gender, age, tumor location, degree of differentiation $(p=0.06)$ and thoracic duct ligation had no significant effect on OS ( $p$ $>0.05$ ), but depth of invasion and presence of cancer embolus had significant effect on OS $(p<0.05)$.

Table II: Single factor analysis of the influence of clinical and pathological factors on DFS and OS.

\begin{tabular}{|c|c|c|c|c|}
\hline Data & Classification & N (\%) & $\begin{array}{l}\text { DFS } \\
\text { p-value }\end{array}$ & $\begin{array}{c}\text { OS } \\
\text { p-value }\end{array}$ \\
\hline \multirow{2}{*}{ Gender } & Male & $57(82.6)$ & \multirow{2}{*}{0.9785} & 0.9797 \\
\hline & Female & $12(17.4)$ & & \\
\hline \multirow{2}{*}{ Age } & $\geq 60$ & $39(56.5)$ & 0.8180 & 0.3687 \\
\hline & $<60$ & $30(43.5)$ & & \\
\hline \multirow{3}{*}{ Location } & Upper & $11(16.0)$ & \multirow{3}{*}{0.4034} & 0.7202 \\
\hline & Middle & $35(50.7)$ & & \\
\hline & Lower & $23(33.3)$ & & \\
\hline \multirow{3}{*}{ Invasion depth } & $\mathrm{T} 1 \mathrm{~b}$ & $33(47.8)$ & 0.0135 & $<0.0001$ \\
\hline & T2 & $22(31.9)$ & & \\
\hline & T3 & $14(20.3)$ & & \\
\hline \multirow{3}{*}{ Differentiation } & G1 & $14(20.3)$ & 0.0204 & 0.0600 \\
\hline & G2 & $39(56.5)$ & & \\
\hline & G3 & $16(23.2)$ & & \\
\hline \multirow{2}{*}{$\begin{array}{l}\text { Cancer } \\
\text { thrombus }\end{array}$} & Yes & $15(21.7)$ & 0.0020 & 0.0055 \\
\hline & No & $54(78.3)$ & & \\
\hline \multirow{2}{*}{$\begin{array}{l}\text { Thoracic duct } \\
\text { ligation }\end{array}$} & Yes & $33(47.8)$ & \multirow{2}{*}{0.0325} & 0.7085 \\
\hline & No & $36(52.2)$ & & \\
\hline
\end{tabular}
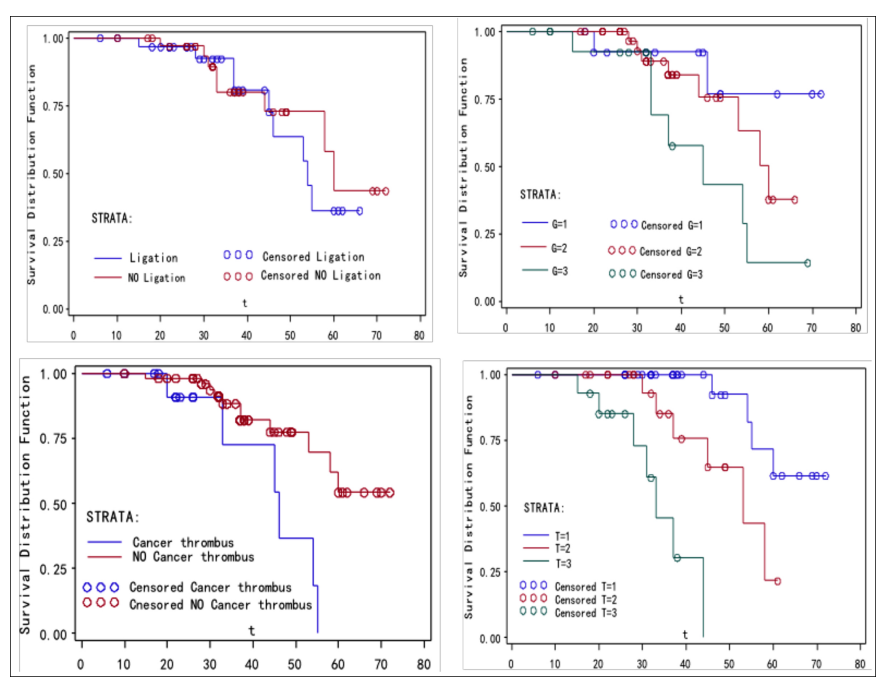

Figure 2: Single factor analysis of the effect of T stage, tumor differentiation, thoracic duct ligation and vasculartumor thrombus on $0 S$.

Cox proportional hazard models for OS was built for those prognostic factors with $p<0.1$ in the univariate analysis as shown in Table III-b. The result show that age $[\mathrm{HR}=0.895,95 \% \mathrm{Cl}(0.819$, $0.977), p=0.0129]$, invasion depth $[\mathrm{HR}=21.968,95 \% \mathrm{Cl}(5.366$, 89.29), $p<0.0001]$, vascular cancer embolus [HR=7.183, $95 \% \mathrm{Cl}$ $(1.700,30.351), p=0.0073$ ] were the independent risk factors affecting the $0 \mathrm{~S}$.

\section{DISCUSSION}

Thoracic duct ligation is an effective method for the treatment of chylothorax, but there are academic disagreements on whether preventive ligation can prevent chylothorax. ${ }^{3-5,15}$ With the development of medicine, people are paying more and more attention to the unlimited potential of immune system in tumor treatment. Previous studies confirmed that preventive thoracic duct ligation had a bad effect on patients, especially the inhibition of postoperative immune function. ${ }^{7,8}$ This time, a long-term follow-up of the patients was conducted to detect early metastasis and calculate survival time.

According to the guidelines, for intramucosal esophageal cancer (Tla), endoscopic rather than thoracoscopic resection is generally chosen ${ }^{9-12}$ At the same time, the patients beyond T3NOMO who often need chemoradiotherapy and then have interference factors on OS and DFS, therefore, TIb-3NOMO patients were selected as the subjects. ${ }^{9-12}$

Some early scholars proposed that in the traditional thoracotomy esophagectomy, preventive thoracic duct ligation will not affect the immune function of patients, so it will ${ }^{13-15}$ not have a significant impact on the long-term survival of patients. Unlike left side transthoracic esophagectomy, thoracoscopic esophagectomy is usually performed through the right chest, and the azygos vein arch usually needs to be ligated and cut off for easier to free esophagus and clean lymphonode. ${ }^{16}$ There are a number of communicating branches between azygos vein and thoracic duct. ${ }^{17}$ Most of the lymph in the whole body flows back to the blood through the thoracic duct or the collateral circulation between the thoracic duct and the azygos vein. When the thoracic duct and azygous arch are transected at the same time, lymph fluid and a large number of lymphocytes in it are difficult to return to the blood in a short time. It can be imagined that the decrease of immune function after operation might give the residual tumor cells an opportunity to escape and metastasize. ${ }^{18}$ In this study, postoperative follow-up also confirmed this result. Cox regression analysis showed that preventive thoracic duct ligation had a significant impact on DFS and was a high-risk factor for postoperative metastasis. The results of this study are the same as those of Fei, ${ }^{19}$ thoracic ductligation did notaffect the OSsignificantly, butit was an independent risk factor affecting DFS.

In the study of the effect of age on long-term survival, the results of univariate analysis and multivariate analysis were different. The authors analysed the reasons as follows: In the KaplanMeier analysis of OS, 60 years was taken as the boundary and changed into a binary variable. The result showed that age $(p=$ 0.3687 ) had no significant effect on OS. In the Cox regression analysis of OS, the age ( $p=0.0129)$ was a continuous variable and entered the regression model and became independent riskfactors of $\mathrm{OS}$.

Table III-a: Cox regression multivariate analysis results of DFS.

\begin{tabular}{|l|l|l|l|l|l|}
\hline DATA & $\boldsymbol{\beta}$ & SE & Wald X2 & p & HR (95\%CI) \\
\hline
\end{tabular}




\begin{tabular}{|l|l|l|l|l|l|}
\hline Invasion depth & 0.91445 & 0.28545 & 10.2627 & 0.0014 & $2.495(1.426,4.366)$ \\
\hline Differentiation & 1.08642 & 0.37315 & 8.4768 & 0.0036 & $2.964(1.426,6.158)$ \\
\hline Cancer embolus & 1.21929 & 0.58371 & 4.3633 & 0.0367 & $3.385(1.078,10.626)$ \\
\hline Thoracic duct ligation & 1.40811 & 0.50959 & 7.6353 & 0.0057 & $4.088(1.506,11.099)$ \\
\hline
\end{tabular}

Table III-b: Cox regression multivariate analysis results of OS.

\begin{tabular}{|l|l|l|l|l|l|}
\hline DATA p & $\boldsymbol{\beta}$ & SE & Wald $\mathbf{2}$ & $\mathbf{p}$ & $\mathbf{H}(\mathbf{9 5} \% \mathbf{C l})$ \\
\hline Age & -0.11148 & 0.04484 & 6.1817 & 0.0129 & $0.895(0.819,0.977)$ \\
\hline Invasion depth & 3.08957 & 0.71910 & 18.4591 & $<0.0001$ & $21.968(5.366,89.926)$ \\
\hline Cancer embolus & 1.97169 & 0.73529 & 7.1905 & 0.0073 & $7.183(1.700,30.351)$ \\
\hline
\end{tabular}

Although in Qiu's study, age as a hierarchical variable also obtained the same results, the present authors believe that in Cox regression analysis, age is more accurately calculated as a continuous variable. ${ }^{20}$

\section{CONCLUSION}

Thoracic duct ligation can affect the DFS after thoracoscopic esophagectomy in esophageal squamous cell carcinoma patients with pT1b-3NOMO stage. The thoracic duct ligation, depth of invasion, degree of differentiation and presence of tumor thrombus are independent factors affecting DFS; meanwhile the depth of invasion, presence of cancer embolus and age were independent factors affecting OS.

\section{ETHICAL APPROVAL:}

The study was approved by the Committee on Medical Ethics of the Hospital.

\section{PATIENTS' CONSENT:}

Written informed consents were obtained from all patients.

\section{CONFLICT OF INTEREST:}

The authors declared no conflict of interest.

\section{AUTHORS' CONTRIBUTION:}

RY: Experimental design, ethical application defence, main surgery, statistical analysis, article writing.

SS: Preoperative and postoperative gastroscopy, biopsy, ultrasonic endoscopy.

LY: Experimental design, article writing.

$\mathrm{HL}$ : Intraoperative anesthesia, postoperative monitoring.

RZ: Operation, personnel coordination, postoperative followup.

PW: Funds management, participation in surgery, postoperative management.

\section{REFERENCES}

1. Si-Yang Liu, Yi-Long Wu. An immunological storm for cancer therapy: 2018 Nobel Prize in Physiology or Medicine. Science Bulletin 2018; 24:1608-10. doi: 10.1016/j.scib. 2018.11.023

2. De AK. Physiology or medicine. Science and Culture 2019; 85:213-4.

3. Miao L, Zhang Y, Hu H, Ma L, Shun Y, Xiang J, et al. Incidence and management of chylothorax after esophagectomy. Thorac Cancer 2015; 6(3):354-8. doi: 10.1111/1759-
7714.12240.

4. Fu JH, Hu Y, Huang WZ, Yang H, Zhu ZH, Zheng B, et al. Evaluating prophylactic ligation of thoracic duct during radical resection of esophageal carcinoma. Ai Zheng 2006; 25(6):728-30.

5. Hou X, Fu JH, Wang X, Zhang LJ, Liu QW, Luo KJ, et al. Prophylactic thoracic duct ligation has unfavorable impact on overall survival in patients with resectable oesophageal cancer. Eur J Surg Oncol 2014; 40(12):1756-62. doi: 10.1016/j.ejso.2014.05.002.

6. Akhtar NM, Chen D, Zhao Y, Dane D, Xue Y, Wang W, et al. Postoperative short-term outcomes of minimally invasive versus open esophagectomy for patients with esophageal cancer: An updated systematic review and meta-analysis. Thorac Cancer 2020; 11(6):1465-75. doi: 10.1111/17597714.13413.

7. Yang RF, Liu TT, Wang P, Zhang RQ, Li C, Han B, et al. Ligation of thoracic duct during thoracoscopic esophagectomy can lead to decrease of T Iymphocyte. J Cancer Res Ther 2018; 14(7): 1535-9. doi: 10.4103/jcrt.JCRT_596_17.

8. Yang RF, Jiang ZM, Zhang RQ, Yu B, Wang XH, Wang P. Effect of ligation of the thoracic duct during oesophagectomy on the absorption of D-xylose. J Coll Physicians Surg Pak 2017; 27(3): 153-6.

9. National health commission of the people's republic of China. Chinese guidelines for diagnosis and treatment of esophageal carcinoma 2018 (English version). Chin J Cancer Res 2019; 31:223-58.

10. Ajani JA, D'Amico TA, Almhanna K, Bentrem DJ, Besh S, Chao $J$, et al. Esophageal and esophagogastric junction cancers, version 2.2015. J Natl Compr Canc Netw 2015; 13:194-227.

11. Liu Huang. Updates in version 2.2018 of the NCCN guidelines for esophageal and esophagogastric junction cancers. Oncol Translational Med 2018; 4:116-9.

12. Ajani JA, D'Amico TA, Bentrem DJ, Chao J, Corvera C, Das P, et al. Esophageal and esophagogastric junction cancers, version 2.2019, NCCN clinical practice guidelines in oncology. J Natl Compr Canc Netw 2019; 17(7): 855-83. doi: 10.6004/jncen.2019.0033.

13. Du ZS, Li XY, Luo HS, Wu SX, Zheng CP, Li ZY, et al. Preoperative administration of olive oil reduces chylothorax after minimally invasive esophagectomy. Ann Thorac Surg 2019; 107(5):1540-3. doi: 10.1016/j.athoracsur.2018.10.053

14. Chew T, Bright T, Price TJ, Watson DI, Devitt PG. Follow-up practices of surgeons and medical oncologists in Australia and New Zealand following resection of esophagogastric cancers. Ann Thorac Cardiovasc Surg 2017; 23(5):217-22. doi: 10.5761/atcs.oa.17-00049. 
15. Glatz T, Marjanovic G, Hoeppner J. Prevention and surgical therapy of chylothorax. Zentralbl Chir 2018; 143(3): 278-83. doi: 10.1055/a-0608-5082.

16. Mao T, Fang W, Gu Z, Guo X, Ji C, Chen W. Video-assisted thoracolaparoscopic esophagectomy: The experience of shanghai chest hospital. J Thorac Dis 2013, 5(6): 906-9. doi: 10.3978/j.issn.2072-1439.2013.12.14.

17. Xiao-wen $Z$, jie $Y$, Wen-guo L, Feng $H$, Yue-jian W, Bin $Z$, et al. Applied anatomic study of video-assisted thoracoscopic ligation of thoracic duct. Chinese J Clin Anatomy 2014; 32:264-8.

18. Onal C, Yildirim BA, Guler OC, Mertsoylu H. The utility of pretreatment and posttreatment lymphopenia in cervical squamous cell carcinoma patients treated with definitive chemoradiotherapy. Int J Gynecol Cancer 2018; 28(8): 1553-9. doi: 10.1097/IGC.0000000000001345.

19. Fei X, Wang X, Lu Q, Lu C, Chen H, Li C. The influence of thoracic duct ligation on long-term survival of patients with esophageal cancer: A propensity score-matched analysis. J Thorac Dis 2020; 12(10): 5532-41. doi: 10.21037/jtd-201341.

20. Qiu MJ, Yang SL, Wang MM, Li YN, Jiang X, Huang ZZ, et al. Prognostic evaluation of esophageal cancer patients with stages I-III. Aging (Albany NY) 2020; 12(14):14736-53. doi: 10.18632/aging.103532. 